\title{
SPENDING ON RHEUMATOID ARTHRITIS AND MORTALITY RATE IN DIFFERENT ADMINISTRATIVE REGIONS OF BRAZIL: IS THERE A RELATIONSHIP?
}

\author{
Bárbara Manfroi', *, Joana Faccioli Japur² \\ 1.Faculdade São Leopoldo Mandic, Campinas (SP), Brazil. 2.Universidade Luterana do Brasil, Canoas (RS), Brazil. \\ *Corresponding author: barbara_manfroi@hotmail.com
}

\section{BACKGROUND}

Rheumatoid arthritis (RA) is a symmetrical, inflammatory and peripheral polyarthritis of unknown etiology. Rheumatoid arthritis affects about $1 \%$ of the world population, making it one of the most common inflammatory arthritis. This pathology is strongly associated with cardiovascular diseases and early mortality, being more lethal in countries with less economic development. Thus, the objective of the study was to correlate spending on rheumatoid arthritis in Brazil with the mortality rate in the different administrative regions of the country.

\section{MATERIALS AND METHODS}

An ecological study was carried out. Data on deaths and expenses with RA were collected on the DATASUS platform for adults over 20 years old, between the years 2008 and 2016. The deaths were stratified by sex, administrative region and age group. Death rate was adjusted by age group. The normality of the data was assessed by the Shapiro-Wilk test and then the Pearson or Spearman test was performed to assess the correlations.

\section{RESULTS}

During the study period, 912 deaths from RA were recorded in DATASUS in individuals over 20 years of age. The region with the highest mortality rate was the Midwest (0.09:100,000 people/year). A positive correlation was observed between the general mortality rate and expenses for RA in Brazil (cor: $0.693, p=0.038$ ). In the analysis of subgroups, a strong and positive correlation was observed between the mortality rate and expenditures in the Southeast (cor: 0.884, $p<0.001$ ) and South (cor: 0.665 , $p=0.049$ ). There was no significant correlation in the other regions or when stratifying by sex and age group. Furthermore, there was no significant correlation between the death rate and the human development index.

\section{CONCLUSION}

In the present study, we observed that in Brazil there is a positive correlation between expenses and mortality rate due to RA and the same was found in regions with greater economic development. A hypothesis suggested is that in these regions there is a greater diagnosis of this disease and, consequently, a greater distribution of resources. It is important, however, that resources also reach less economically favored regions, in order to provide better treatment for patients with RA and thus increase survival. 